\title{
Community Detection of Chinese Micro-Blogging Using Multi-Dimensional Weighted Network
}

\author{
Xiaoping Zhou ${ }^{1,2,}$, Xun Liang ${ }^{1}$ and and Run $\mathrm{Cao}^{1}$ \\ ${ }^{1}$ Renmin University of China, Beijing 100782, China; ${ }^{2}$ Beijing University of Civil Engineering and Architecture, Bei- \\ jing, 100040, China
}

\begin{abstract}
Existing community detection methods are mostly based on the analysis of the links among the nodes, ignoring the rich, while the others often ignore the network structure which is the foundation of social media. Aiming at the existed problems, this paper proposed a community detection algorithm based on multi-dimensional weighted network. By introducing User Interactive Frequency, User Interest Similarity, and User Attributes Similarity into the basic network topology, a multi-dimensional weighted network is set up. After converting the multi-dimensional weighted network into a single-dimensional weighted network, an improved CNM algorithm is exploited to discover the communities. A corresponding series of evaluation indicators are proposed to evaluate the detection results. By evaluating the algorithm in the dataset of Chinese Micro-blogging, it reveals that the clustering results are better when extra information is used, and in Chinese Micro-blogging platform, User Interactive Frequency plays a much more important role in community detection.
\end{abstract}

Keywords: Chinese, CNM, community detection, micro-blogging, multi-dimensional, weighted network.

\section{INTRODUCTION}

Community can refer to a usually small, social unit of any size that shares common values. Since the advent of the Internet, the concept of community has less geographical limitation. Micro-blogging is a broadcast medium in the form of blogging. With hundreds of millions of people gathering virtually in a Micro-blogging, such as Facebook and sina Weibo (the most widely used Chinese Micro-blogging), it has been a typical online virtual community.

With the development of $3 \mathrm{G}$ technology and the gradual improvement of mobile access to the Internet, both the user scale and social influence of Micro-blogging sites have experienced rapid growth. Currently, Micro-blogging is becoming a widely-accepted platform to express people's views and opinions. By its application in communication, marketing, political purposes and so on, Micro-blogging plays important roles in our everyday life. Thus, the research results of community detection on Micro-blogging platform have practical significance, which can be used to guide the network marketing, public opinion control and many other issues. Essentially, for its hybrid network and the aspects of both social networks and information networks, Microblogging network is a complex network.

Community detection, which is fundamental for further researches on community in complex network, has gained of paramount attentions. As a representative complex network, Micro-blogging attracts a number of researches, some of which are working on community detection.
Existing community detection methods are mostly based on the analysis of the links between nodes and ignore the rich information [1-4] of new era social media, while the others $[5,6]$ often ignore the network structure which is the foundation of social media. With the continuous expansion of the research on complex network, especially on online communities, some scholars tried to apply the content of nodes and edges of the network to the community detection. Yan et al. proposed a community detection method, which integrated the interest and network topology [7]. Java et al. synthesized the links and the labels in folksonomy, proposed a novel community detection method, and got a more precise community classification [8]. Zhang et al. combined user friendship network and user-generated content, used NMF and author-topic AT model, and gained a better result in Delicious and Twitter dataset, thus proposing a method to detect the interest-based community by finding out the leading user in certain interest field.

The Micro-blogging contains a variety of links, vast amounts of user generated content, and a large number of user attribute metadata information which can be used for user clustering. Though, these data are from different dimensions, they are substantial for Micro-blogging platform which has sociability and media. And in Micro-blogging community detection, all of the related information should be taken into consideration.

This paper aims to introduce the related information into the basic Micro-blogging network topology, and gets a better community detection result.

The rest of the paper is organized as follows. The literature review is presented in Section 2. Section 3 presents in detail the problem definition and the framework. Section 4 introduces the detailed algorithm. Section 5 introduces a 
Table 1. Comparison of classic community detection algorithms.

\begin{tabular}{|c|c|c|c|}
\hline Algorithms & Time Complexity & Pros & Cons \\
\hline \hline $\begin{array}{c}\text { Spectral Bisection } \\
\text { Method }\end{array}$ & $O\left(n^{3}\right)$ & Fast & Fast \\
\hline $\begin{array}{c}\text { Kemighan-Lin Algo- } \\
\text { rithm }\end{array}$ & $O\left(t n^{2}\right)$ & $\begin{array}{c}\text { Priori knowledge (e.g., the number or the aver- } \\
\text { age scale of the communities) is required, and } \\
\text { can't be apply to real network }\end{array}$ \\
\hline $\begin{array}{c}\text { W-H Algorithm } \\
\text { GN Algorithm }\end{array}$ & $O(n)$ & $\begin{array}{c}\text { Can detect which community the specific node } \\
\text { belongs to, without the details of the whole commu- } \\
\text { nity network structure }\end{array}$ & $\begin{array}{c}\text { Partial information of the network structure is } \\
\text { required }\end{array}$ \\
\hline Newman Algorithm & $O((m+n) n)$ & $\begin{array}{c}\text { The number and the scale of division communities } \\
\text { is not required, and precise }\end{array}$ & $\begin{array}{c}\text { The evaluation of the reasonable number of } \\
\text { division communities is not given, and high } \\
\text { time complexity }\end{array}$ \\
\hline CNM Algorithm & $O\left(n \log ^{2} n\right)$ & $\begin{array}{c}\text { Flose to linear in time complexity, and space com- } \\
\text { plexity is shortened by using the heap structure }\end{array}$ & Suboptimum solution \\
\hline
\end{tabular}

corresponding series of evaluation indicators. Experiments are presented and analyzed in Section 6. Finally, Section 7 concludes the paper with a summary of the future work.

\section{LITERATURE REVIEW}

In recent years, community detection has experienced a rapid development, and becomes significant research field in data mining. Though, a mass of researches and extensive discuss had launched; till now, there's no common method to definite a reasonable community structure of a given network.

Currently, community detection methods on Microblogging can be divided into 2 groups: clustering algorithm and link-based algorithm.

\subsection{Clustering Algorithm}

Clustering algorithm, which requires information or properties of the network except for the space topology, consists of traditional clustering algorithm (such as K-Mean Algorithm, DBSCAN Algorithm, and so on), text clustering algorithm (such as Latent Dirichlet Allocation Model, Vector Space Model, TF-IDF Model), and the algorithms derived from the above.

\subsection{Link-based Algorithm}

In some complex networks, it's difficult or cost-toomuch to acquire information or properties. In these scenarios, only can the basic links between the nodes be used, and the community detection methods include traditional graphic-based algorithm (such as Kernighan-Lin Algorithm), spectral analysis clustering algorithm (such as Spectral Bisection Method, Unnormalized Spectral Clustering, and Normalized Spectral Clustering), splitting algorithm (such as GN algorithm) and aggregation algorithm (such as CNM algorithm).
As the only handful resource is the relations among the nodes in the primary network, how to treat the relations respectively in community detection is becoming a hot research problem. Weighting the edge of the network, which is proposed by M. Girvan and M.E.J. Newman in GN algorithm, has been extensive used. In GN algorithm, the edge betweenness is introduced to evaluate the influence of edge in the network. By removing the edge with the largest edge betweenness iteratively, it divides a huge network into small ones. GN algorithm, which has been proved in community detection, is limited in the network with small scale for its high costs in calculating. To solve the issue, some other fast algorithms were proposed, such as Newman algorithm, CNM algorithm. Table 1 shows the comparison of classic community detection algorithms.

\section{PROBLEM DEFINITION}

The related information (e.g., a variety of links, vast amounts of user generated content, and a large number metadata of user attribute information) in a Micro-blogging is taken into account to obtain a better community classification. And the terminologies related to the work should be defined, and the overall solution framework should be given.

\subsection{Terminology Definition}

Currently, the research on online community structure is mainly on the detection tragedy and method. Micro-blogging is an online community with huge number of participants. And it can be defined in relation network's way.

Definition 1. Micro-blogging Topology Graph

Micro-blogging Topology Graph can be defined as: $G=(V, E, W)$

where $G$ is a weighted digraph, $V$ is the set of nodes, $E$ is the set of directed edges, $W$ is the set of the weights of the edges. 
Define $V$ as $V=\left\{v_{1}, v_{2}, \ldots, v_{n}\right\}$, and $v_{i} \in V$ represents a Microblogging user. Define $E$ as $E=\left\{e_{i j} \mid e_{i j}=\left(v_{i}, v_{j}\right)\right.$, and $e_{i j} \in E$ represents the relationship between user $v_{i}$ and user $v_{j}$, and $v_{i}$, $v_{j} \in V$. Define $W$ as $W=\left\{w\left(e_{i j}\right) \mid \forall e_{i j} \in E\right\}$, and $w_{i j}$ represents the stength of the relation $e_{i j}$.

In Chinese Micro-blogging (sina Weibo), one user can follow any other user. And the follower can receive all the content the followee generated. Based on the follow relationship, a basic network, called follower-followee network, is built. Moreover, the follower-followee network can subdivide to two sub-networks. Only when both user $v_{i}$ follow user $v_{j}$ and user $v_{j}$ follows user $v_{i}$ could the relation $e_{i j}$ is established. In this situation, the network is defined as Microblogging Bi-followed Network. In some other scenarios where the relation $e_{i j}$ is set either when user $v_{i}$ follow user $v_{j}$ or when user $v_{j}$ follows user $v_{i}$, the network is called Microblogging Single-followed Network.

Both Micro-blogging Bi-followed Network and Microblogging Single-followed Network are basic Micro-blogging networks. As Micro-blogging Bi-followed Network has a stricter construction condition, it's a sub network of Microblogging Single-followed Network, and has a sparse relationship. Needless to say, the strength of relation can be added to either network.

\section{Definition 2. User Interactive Frequency}

In Chinese Micro-blogging (sina Weibo), a user can forward another user's micro-blog or mention some other users in his micro-blog. User Interactive Frequency is defined to show how many times one user forward or mention another one. And User Interactive Frequency can be defined as:

$F=\left\{f\left(e_{i j}\right) \mid \forall e_{i j} \in E\right\}$

where $f\left(e_{i j}\right)$ indicates the interaction between user $v_{i}$ and user $v_{j}$. In both Micro-blogging Bi-followed Network or Microblogging Single-followed Network, $f\left(e_{i j}\right)$ equals to the sum of the times user $v_{i}$ mentioned or forwarded user $v_{j}$ and user $v_{j}$ mentioned or forwarded user $v_{i}$. Obviously, $F$ is a type of the strength for relation $e_{i j}$, and it is a certain type of $W$.

\section{Definition 3. User Interest Similarity}

To a certain extent, what a user published in his microblog reflects what he is interesting in. And by analyzing the content a user generated, the User Interest Model can be calculated. Define the users' interest model as:

Interest $_{i}=\left\{\right.$ Word $_{1}$, Word $_{2}, \ldots$, Word $\left._{n}\right\}$

where Interest $t_{i}$ means the User Interest Model of user $v_{i}$. Apparently, one user is always interesting in a series of things. And each keyword Word $_{i}$ implies one item he cares. Moreover, user places distinct emphasis on each keyword. In other words, each keyword has its own interest weight. And Word can be described as:

Word $_{i}=\left(\right.$ Keyword $_{i}$, Weight $\left.{ }_{i}\right)$

Thus, the problem is converted to how to find out the keywords and their weights.

Different from English content, where all the words are separated naturally by character 'space', Chinese content should be segmented to find out the words. In this paper, Chinese segmentation system ICTCLAS, which is developed by Chinese Academic of Science, is used to gain the keywords.

After the Chinese word segmentation, TF-IDF is exploited to calculate the weight of each keyword. By ranking the keywords in the order of their TF-IDF value, top $20 \%$ keywords are selected into the users' User Interest Model.

By analyzing the content published by user, User Interest Model is obtained. User Interest Similarity is defined to described how much is the similarity in interest between two users in a Micro-blogging. And it can be defined as:

$C=\left\{c\left(e_{i j}\right) \mid \forall e_{i j} \in E\right\}$

where $c\left(e_{i j}\right)$ means the interest similarity between user $v_{i}$ and user $v_{j}$.

From each user's User Interest Model Interest ${ }_{i}$, it can be treated as a vector in geometry. And Vector Space Model (VSM) is easily coming out to solve the problem. By using VSM, $c\left(e_{i j}\right)$ can be simply calculated by the following formula.

$$
\begin{gathered}
c\left(e_{i j}\right)=\operatorname{sim}_{\left(\text {Interest }_{i}, \text { Interest }_{j}\right)} \\
=\frac{\sum_{k=1}^{n} \text { Weight }_{i k} \text { Weight }_{j k}}{\sqrt{\sum_{k=1}^{n} \text { Weight }_{i k}^{2} \times \sum_{k=1}^{n} \text { Weight }_{j k}^{2}}}
\end{gathered}
$$

where Weight $_{i k}$ means the weight of Word $_{k}$ in user $v_{i}$ 's User Interest Model. Only the same keywords in User Interest Model are calculated in the formula. Undoubtly, $C$ is another typical $W$.

\section{Definition 4. User Attribute Similarity}

In Chinese Micro-blogging (sina Weibo), User's basic information includes his location (province and city), gender, number of fans, number of friends, registration time and so on. And all of these data are significant for community detection of Micro-blogging. The location (province and city) implies the user's geographic position; the number of fans shows the influence in the network; the number of blogs shows the user's activity; the registration time shows his level in Micro-blogging. And User Attribute Model can be described as:

$$
\begin{aligned}
& \text { Attr }=\{\text { gender }, \text { createdtime, followers, friends, } \\
& \text { biFollowers, statuses, verified, province, city }\}
\end{aligned}
$$

$A t t r_{i}$ represents the User Attribute Model of user $v_{i}$. Similarly, User Attribute Similarity can be defined as:

$A=\left\{a\left(e_{i j}\right) \mid \forall e_{i j} \in E\right\}$

where $a\left(e_{i j}\right)$ means the interest similarity between user $v_{i}$ and user $v_{j}$.

Then VSM is utilized to calculate the User Attribute Similarity.

where $w_{i k}$ means the value of the corresponding attribute of Attr $_{i}$. Also, $A$ belongs to $W$.

$$
a\left(e_{i j}\right)=\operatorname{sim}\left(\text { Interest }_{i}, \text { Interest }_{j}\right)
$$




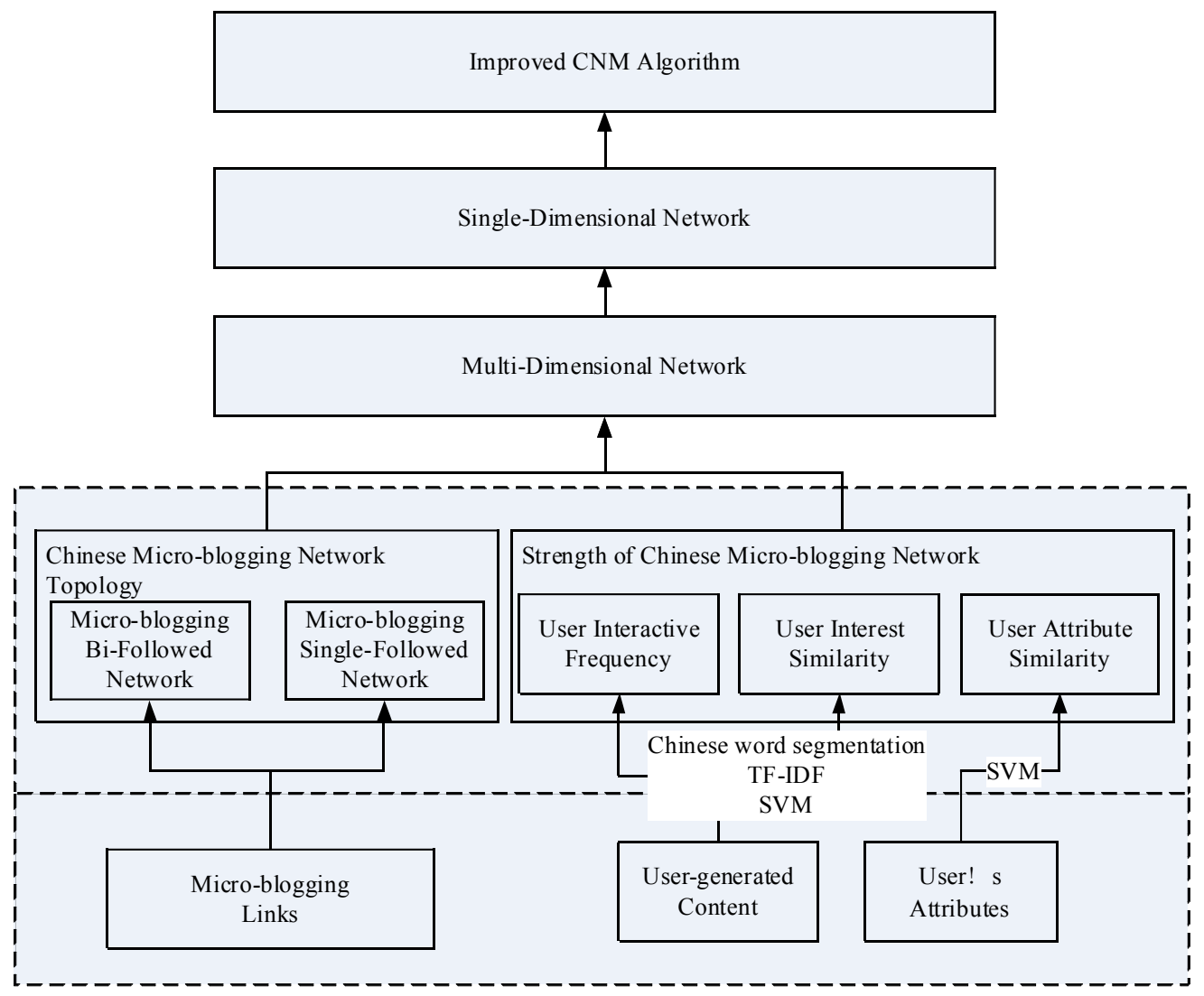

Fig. (1). Overall solution framework.

$$
=\frac{\sum_{k=1}^{n} w_{i k} w_{j k}}{\sqrt{\sum_{k=1}^{n} w_{i k}^{2} \times \sum_{k=1}^{n} w_{j k}^{2}}}
$$

\subsection{Overall Solution Framework}

As shown in Fig. (1), this paper builds the basic network topology through Micro-blogging links. It collects 7776 users with their attributes and user-generated content of sina Weibo. By analyzing the data, Micro-blogging Singlefollowed Network is formed, which is the basic for Microblogging Bi-followed Network. Users' attributes are used to calculate the User Attribute Similarity using SVM. User Interactive Frequency is extracted from the user-generated content. User Interest Similarity is worked out after three steps: Chinese word segmentation, TF-IDF and VSM.

Based on the basic network and the strength, multidimensional network is set up. And the community detection algorithm stated in section 4 is used to convert the multidimensional network into a single-dimensional network, where classic community detection algorithms can be used.

\section{COMMUNITY DETECTION ALGORITHM}

Aiming at the "complex" characteristics of Chinese Micro-blogging (sina Weibo), this paper proposes an community detection algorithm based on weighted networks. Combing the three typical edge betweennesses (User Interactive Frequency $F$, User Interest Similarity $C$, and User Attribute
Similarity $A$ ) to either of the two basic Micro-blogging networks brings forth three single-dimensional networks. Each time when a new edge betweenness is added to the basic network, it generates a new single-dimensional network. As all these single-dimensional networks are based on the same basic network topology graphic, they can be treated as a multi-dimensional weighted network. On this point, the varieties of the information in Micro-blogging generate the multi-dimensional weighted network.

Considering a p-dimensional network as:

$$
\hat{A}=\left\{A^{(1)}, A^{(2)}, \cdots, A^{(p)}\right\}
$$

where $A^{(i)} \in\{0,1\}^{n \times n}$ means the interactive status in the $\mathrm{i}$-th dimension.

As there will exist kinds of potential interactive methods in a Micro-blogging, the underlying shared community structure will be extremely complicated, which make it necessary to integrate the multi-dimensional network to find out the shared community structure.

A valid quomodo to process the multi-dimensional network is to convert it into a single-dimensional network. From the perspective of set theory, a variety of interactive forms among nodes strengthen their relation. And a multidimensional network can be transformed to:

$$
\bar{A}=\sum_{i=1}^{p} \theta_{i} A^{(i)}
$$


where $\theta_{i}$ signifies the importance of the $i$-th dimension network in the $p$-dimensional network. And often, there exists:

$$
\sum_{i=1}^{p} \theta_{i}=1
$$

Sometimes, $\theta_{i}$ is set to be $1 / p$.

Using $\bar{A}$, community detection in multi-dimensional network is converted to a simple one-dimensional network, where the classic community detection algorithms can be applied. And an improved CNM algorithm is used in this paper.

\section{EVALUATION INDICATORS}

In order to meet the needs of specific scenario, kinds of community detection algorithms are founded. Meanwhile, a series of evaluation tragedies are formed to evaluate the detection results. In this section, a new evaluation tragedy is proposed correspondingly.

For any community, users and topics are two main elements. And the evaluation indicators for the detection result should consider both the users and topics. As Modularity $Q$, which is proposed by Newman in CNM algorithm, has been proved, it's also selected in the evaluation system and named Qs. Moreover, some other indicators related to users or/and topics are introduced.

\section{Definition 5. User Interactive Frequency Indicator}

User Interactive Frequency Indicator is defined to describe the level of activity during communities. A good community classification should result in active interaction during communities. And User Interactive Frequency Indicator is defined as:

$$
\text { Frequency }=\frac{\sum_{i=1}^{n-1} \sum_{j=i+1}^{n} \sum_{k=1}^{\gamma} R_{k i} R_{k j} f\left(e_{i j}\right)}{\sum_{i=1}^{n-1} \sum_{j=i+1}^{n} \sum_{k=1}^{\gamma} R_{k i} R_{k j}}
$$

where $R_{k i}=1$ when user $i$ belongs to the community $k$, otherwise $R_{k i}=0$.

Undoubtedly, good community detection result has nice Frequency.

\section{Definition 6. User Interest Similarity Indicator}

The users in the same community should have many similar interests. And User Interest Similarity Indicator is proposed to indicate the similarity during communities. The definition is:

$$
\text { ContentSu }=\frac{\sum_{i=1}^{n-1} \sum_{j=i+1}^{n} \sum_{k=1}^{\gamma} R_{k i} R_{k j} c\left(e_{i j}\right)}{\sum_{i=1}^{n-1} \sum_{j=i+1}^{n} \sum_{k=1}^{\gamma} R_{k i} R_{k j}}
$$

where $R_{k i}=1$ when user $i$ belongs to the community k, otherwise $R_{k i}=0$.

\section{Definition 7. User Attribute Similartity Indicator}

It is supported that most users have the same attributes, such as living in the same city, having the same age range, and so on. And User Attribute Similarity Indicator is introduced as below:

$$
u s e r S u=\frac{\sum_{i=1}^{n-1} \sum_{j=i+1}^{n} \sum_{k=1}^{\gamma} R_{k i} R_{k j} a\left(e_{i j}\right)}{\sum_{i=1}^{n-1} \sum_{j=i+1}^{n} \sum_{k=1}^{\gamma} R_{k i} R_{k j}}
$$

where $R_{k i}=1$ when user $i$ belongs to the community $k$, otherwise $R_{k i}=0$.

\section{Definition 8. Comprehensive Indicator}

In order to balance the density and similarity in divided communities, a Comprehensive Indicator $\bar{\mu}$ is used and calculated as the average of Frequency, ContentSu, UserSu and Qs.

$$
\bar{\mu}=\frac{Q_{s}+\text { Frequency }+ \text { Content } S u+U \text { serSu }}{4}
$$

In the evaluation system, a higher Qs indicates closer relationship among the users in the community, larger value of Frequency means a larger average number of interaction (mention and forward), bigger number of ContentSu shows the more similar interest of the users and the content, and greater UserSu presents the higher similarity of users' attributes in community. For a good community division, users should have closed relations and share more contents and interest. And it can be evaluated by the indicators stated above.

\section{EXPERIMENTS}

Based on the two basic networks (Micro-blogging BiFollowed Network and Micro-blogging Single-Followed Network) and three weighted methods (User Interactive Frequency, User Interest Similarity, and User Attributes Similarity), 8 experiments, which are divided into 2 groups according to the basic network, are designed to evaluate the community detection results. The former 4 experiments are based on Micro-blogging Single-Followed Network, and the rest on Micro-blogging Bi-Followed Network.

All the experiments use the same data: the same users, the same user relations. The experiment primary network has 1371 users, selected from 7776 users from Chinese Microblogging (sina Weibo) who has at least 50 fans.

As shows in Table 2, no extra information is introduced in Exp. 1, which means all the weights of the edges in Exp.1 are set 1; Exp. 2 uses User Interactive Frequency as the strength of the edges; Exp. 3 combines User Interest Similarity and User Attributes Similarity to the edges using multidimensional network algorithm stated above. Exp. 4 takes all the three factors into the consideration. 
Table 5. Community detection results.

\begin{tabular}{|c|c|c|c|c|}
\hline Indicator & Exp. 5 & Exp. 6 & Exp. 7 & Exp. 8 \\
\hline \hline Qs & 0.5427 & $\mathbf{0 . 6 7 2 7}$ & 0.6100 & 0.6540 \\
\hline Frequency & 2.5589 & 2.7697 & 2.6286 & $\mathbf{2 . 7 9 4 9}$ \\
\hline ContentSu & 0.0398 & 0.0407 & 0.0517 \\
\hline UserSu & 0.4329 & $\mathbf{0 . 4 3 6 5}$ & 0.93517 & 0.4352 \\
\hline $\boldsymbol{\mu}$ & 0.8936 & 0.9799 & 87.200 & $\mathbf{0 . 9 8 4 0}$ \\
\hline Time(s) & 93.316 & 88.348 & 87.794 \\
\hline
\end{tabular}
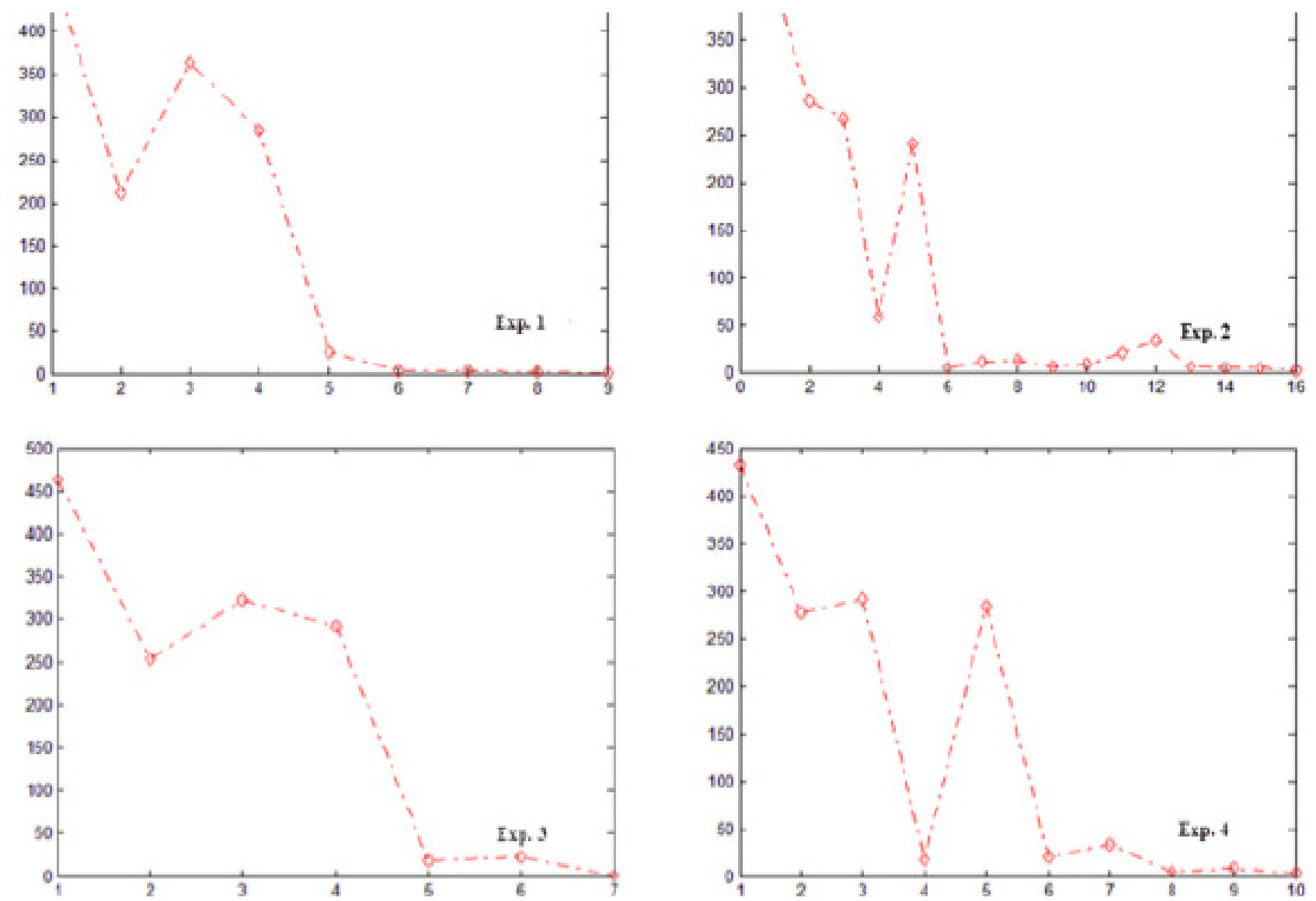

Fig. (2). Community detection results in micro-blogging single-followed network.

periments, but better than the counterpart in Micro-blogging Single-Followed Network. And it indicates better results in Micro-blogging Bi-Followed Network than Micro-blogging Single-Followed Network with the same strength conditions. Exp. 6 and Exp. 8 have the better value in Qs, Frequency and UserSu. And Exp. 6 values highest in UserSu. All of these means User Interactive Frequency is also a key strength in Micro-blogging Bi-Followed Network.

Furthermore, Exp. 8 got the highest score in $\bar{\mu}$, which indicates the comprehensive community detection quality of the result. It illustrates that in Micro-blogging Bi-Followed Network, more information will result in better community detection results.

Fig. (2) shows the number of division communities and the scale of each community in Micro-blogging SingleFollowed Network. X-axis indicates the index of the divided communities, while $y$-axis indicates the scale of the communities. Exp. 1 divided the primary network into 9 communities, while Exp. 2 has 16 communities, Exp. 3 has 7 communities and Exp. 4 has 10 communities. It reveals that the di- 

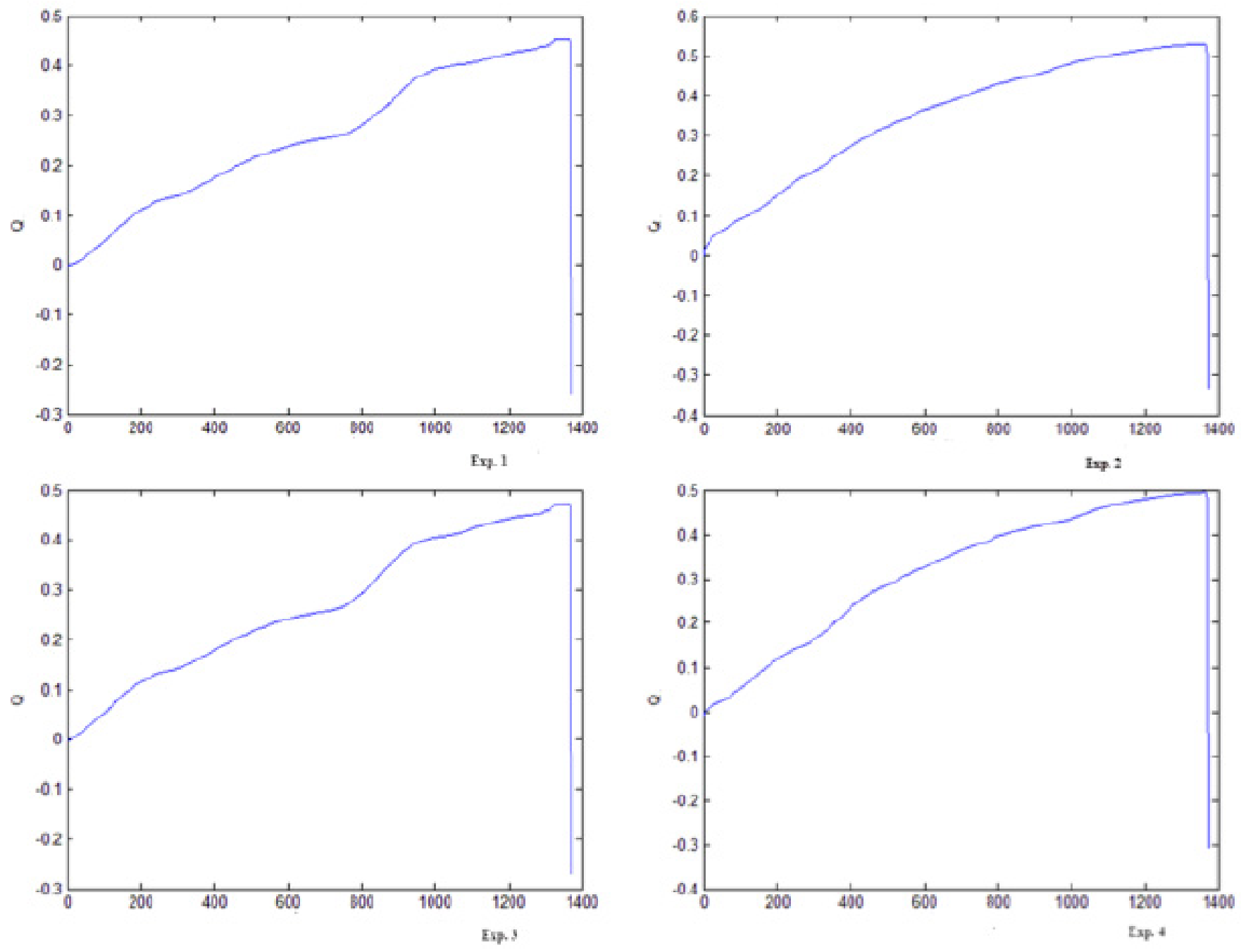

Fig. (3). Community detection results in micro-blogging single-followed network.

vision results turned to be balance in Exp. 2, 3, and 4, and User Interactive Frequency is a strict division factor, which increases the number of division communities. The introduction of User Attributes/Interest Similarity makes the closer relationship in the same community, and reduces the number of the division communities. Exp. 4 balances the number and the scale of the communities.

Fig. (3) shows the community detection results in Microblogging Single-Followed Network. X-axis shows the iterations (the number of the users in the experiment); while $y$ axis shows the Modality $Q s$.

Fig. (4) shows the experiments' results in Microblogging Bi-Followed Network. And it's a lot different from the results in Micro-blogging Single-Followed Network. With the same strength of edges, experiments in Microblogging Bi-Followed Network got much more communities, which is around 2 to 3 times, than that in Microblogging Single-Followed Network. It testifies to the truth that a stricter formed network makes the longer distance among users, and the weak ties are neglected; finally it bring forth more division communities.
While Exp.6 has 23, Exp. 7 has 29 and Exp. 8 has 21. Easily seen from the Fig. (4), Exp. 5 and Exp. 7 don't obtain balance classification results, while the results in Exp. 6 and Exp. 8 seem much better. Different from Micro-blogging Single-Followed Network, User Interactive Frequency aggregates the users in Micro-blogging Bi-Followed Network, and the User Attribute/Interest Similarity makes an increase in the number of division communities.

Fig. (5) shows the community detection results in Microblogging Bi-Followed Network. X-axis shows the iterations (the number of the users in the experiment); while y-axis shows the Modality $Q s$.

\section{CONCLUSION}

Existing community detection methods are mostly based on the analysis of the links between nodes ignoring the rich information of new era social media, the others clustering methods sometime ignore the network structure which is the foundation of social media. Aiming at the existed problems on community detection of Micro-blogging, this paper proposed a community detection algorithm based on multidimensional weighted network. 

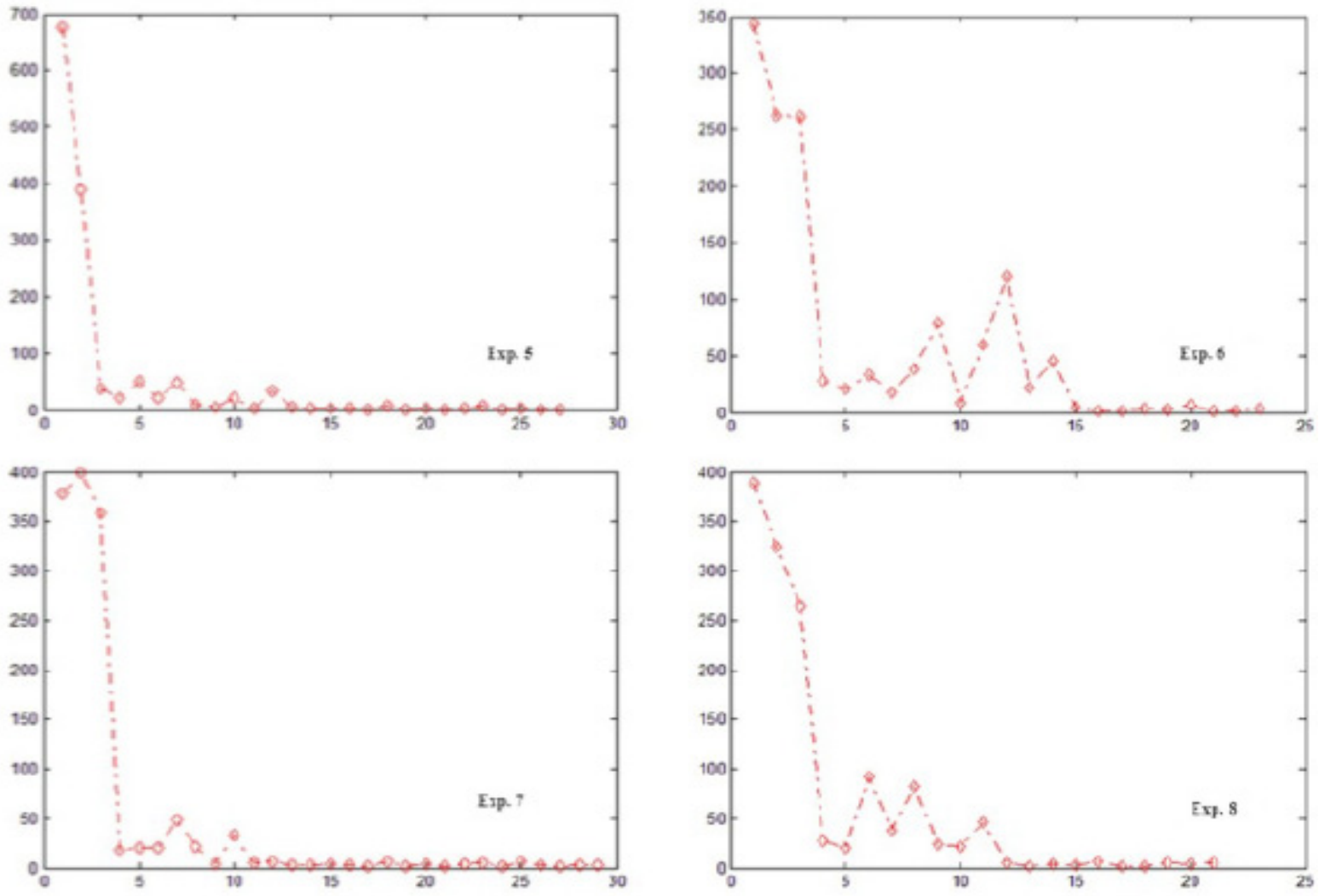

Fig. (4). Community detection results in micro-blogging bi-followed network.
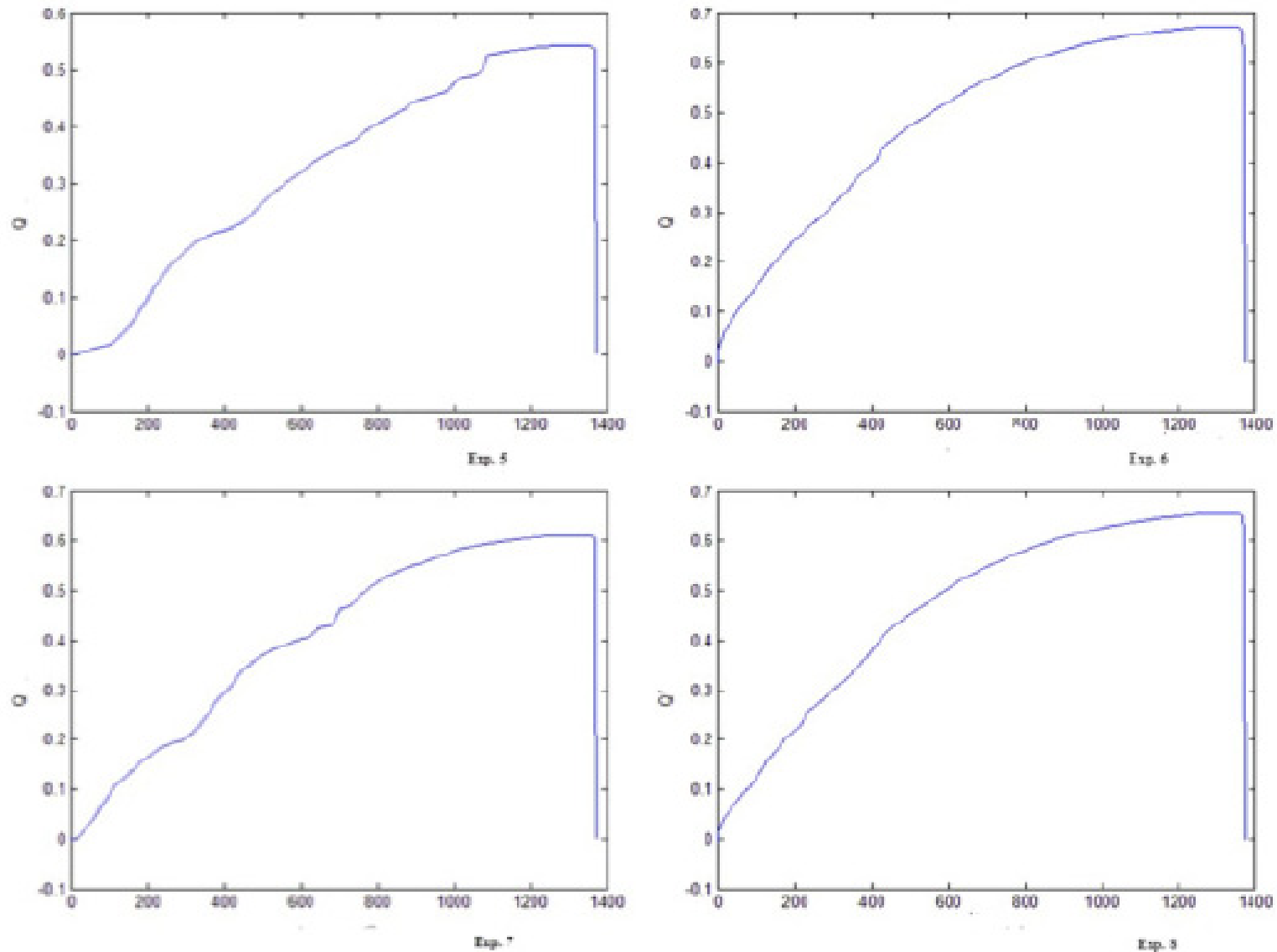

Fig. (5). Community detection results in micro-blogging bi-followed network. 
Firstly, two basic network topologies of Chinese Microblogging are introduced, which are called Micro-blogging Bi-Followed Network and Micro-blogging Single-Followed Network. Secondly, the weighting methods are stated, and three edge betweennesses are defined: User Interactive Frequency, User Interest Similarity, and User Attributes Similarity. Combing the edge betweennesses with the basic network forms a multi-dimensional network. Then, an algorithm, which converts the multi-dimensional network into a single-dimensional network, is proposed.

Also, this paper constructed an comprehensive evaluation indicator $\bar{\mu}$, which is the mean value of the moudulity $Q s$, user interactive frequency within the community Frequency, user interest similarity within the community ContentSu, user attribute similarity within the community UserSu.

The Experimental results show that the classification results are better when extra information is used. And for Chinese Micro-blogging platform, the User Interactive Frequency plays a much more important role in community detection than user Interest Similarity and User Attribute Similarity.

\section{CONFLICT OF INTEREST}

The authors confirm that this article content has no conflict of interest.

\section{ACKNOWLEDGEMENTS}

The research was supported by Beijing Higher Education Young Elite Teacher Project (No. YETP1660), the Natural
Science Foundation of Beijing under grant no. 4132067 and the Natural Science Foundation of China under grant no. 71271211.

\section{REFERENCES}

[1] M. E. J. Newman, and M. Girvan, "Finding and evaluating community structure in networks," Physical Review E, vol. 69, no. 2, pp. 026113, 2004.

[2] S. White, and P. Smyth, "A spectral clustering approach to finding communities in graph," Proceedings of the Fifth SIAM International Conference on Data Mining, pp. 274-285. 2005.

[3] G. Palla, I. Derényi, I. Farkas, and T. Vicsek, "Uncovering the overlapping community structure of complex networks in nature and society", Nature, vol. 435, pp. 814-818, 2005.

[4] X. He, H. Zha, C. H. Q. Ding, and H. D. Simon, "Web document clustering using hyperlink structures," Computational Statistics and Data Analysis, vol. 41, no. 1, pp. 19-45, 2002.

[5] M. Steyvers, P. Smyth, M. Rosen-Zvi, and T. Griffiths, "Probabilistic author-topic models for information discovery," The proceeding of the Thirteenth ACM SIGKDD International Conference on Knowledge Discovery and Data Mining, pp. 306-315, 2004.

[6] D. Zhou, I. Councill, H. Zha, and C. Giles, "Discovering tempora communities from social network documents," Proceedings of the 7th IEEE International Conference on Data Mining, pp. 745-750, 2007.

[7] F. Yan, M. Zhang, Y. W. Tan, J. Tang, and Z. H. Deng, "Community discovery based on actors'interests and social network structure," Journal of Computer Research and Development, vol. 47, pp. $357-362,2010$

[8] A. Java, X. D. Song, T. Finin, and B. Tseng, "Why We Twitter: Understanding Microblogging Usage and Communities," Proceedings of the 9th WebKDD and 1st SNA-KDD 2007 workshop on Web mining and social network analysis, pp. 56-65, 2007.

[9] Z. F. Zhang, Q. D. Li, D. Zeng, and H. Gao, "User community discovery from multi-relational networks," Decision Support Systems, vol. 54, no. 2, pp. 870-879, 2013.

\author{
Received: September 16, 2014 \\ Revised: December 23, 2014 \\ Accepted: December 31, 2014
}

(C) Zhou et al.; Licensee Bentham Open.

This is an open access article licensed under the terms of the (https://creativecommons.org/licenses/by/4.0/legalcode), which permits unrestricted, noncommercial use, distribution and reproduction in any medium, provided the work is properly cited. 